\title{
Gaia DR2 white dwarfs in the Hercules stream
}

\author{
Santiago Torres ${ }^{1,2}$, Carles Cantero ${ }^{1}$, María E. Camisassa ${ }^{3,4}$, Teresa Antoja $^{5}$, Alberto Rebassa-Mansergas ${ }^{1,2}$, \\ Leandro G. Althaus ${ }^{3,4}$, Thomas Thelemaque ${ }^{6}$, and Héctor Cánovas ${ }^{7}$ \\ ${ }^{1}$ Departament de Física, Universitat Politècnica de Catalunya, c/Esteve Terrades 5, 08860 Castelldefels, Spain \\ e-mail: santiago.torres@upc.edu \\ 2 Institute for Space Studies of Catalonia, c/Gran Capità 2-4, Edif. Nexus 104, 08034 Barcelona, Spain \\ 3 Facultad de Ciencias Astronómicas y Geofísicas, Universidad Nacional de La Plata, Paseo del Bosque s/n, \\ 1900 La Plata, Argentina \\ 4 Instituto de Astrofísica de La Plata, UNLP-CONICET, Paseo del Bosque s/n, 1900 La Plata, Argentina \\ 5 Institut de Ciències del Cosmos, Universitat de Barcelona (IEEC-UB), Martí i Franquès 1, 08028 Barcelona, Spain \\ ${ }^{6}$ Industrial and Informatic Systems Deparment, EPF - École d'Ingénieurs, 21 boulevard Berthelot, 34000 Montpellier, France \\ 7 European Space Astronomy Centre (ESA/ESAC), Operations Deparment, Villanueva de la Cañada, 28692 Madrid, Spain
}

Received 5 July 2019 / Accepted 7 August 2019

\begin{abstract}
Aims. We analyzed the velocity space of the thin- and thick-disk Gaia white dwarf population within $100 \mathrm{pc}$ by searching for signatures of the Hercules stellar stream. We aimed to identify objects belonging to the Hercules stream, and by taking advantage of white dwarf stars as reliable cosmochronometers, to derive a first age distribution.

Methods. We applied a kernel density estimation to the $U V$ velocity space of white dwarfs. For the region where a clear overdensity of stars was found, we created a 5D space of dynamic variables. We applied a hierarchichal clustering method, HDBSCAN, to this 5D space, and identified those white dwarfs that share similar kinematic characteristics. Finally, under general assumptions and from their photometric properties, we derived an age estimate for each object.

Results. The Hercules stream was first revealed as an overdensity in the $U V$ velocity space of the thick-disk white dwarf population. Three substreams were then found: Hercules $a$ and Hercules $b$, formed by thick-disk stars with an age distribution that peaked 4 Gyr in the past and extends to very old ages; and Hercules $c$, with a ratio of 65:35 of thin to thick stars and a more uniform age distribution that is younger than $10 \mathrm{Gyr}$.
\end{abstract}

Key words. white dwarfs - Galaxy: kinematics and dynamics - solar neighborhood - methods: data analysis

\section{Introduction}

Stars from the solar neighborhood (the volume of the Galaxy up to a few hundred parsec from the Sun) are far from presenting a uniform and homogeneous velocity distribution. In addition to the Galactic components of the thin and thick disk and the stellar halo, several kinematic structures left their imprint on the velocity space. The origin of these structures is intensely debated and involves a wide variety of hypotheses ranging from non-axisymmetric structural components (such as the bar and the spiral arm of the Galaxy) to cluster disruption or past accretion events (for a thorough review, see Antoja et al. 2010). In any case, the origins represent relevant signatures of the structure and dynamical evolution of the Galaxy.

One of the most prominent kinematic features among them is the Hercules stream, also known as the $U$-anomaly. The Hercules stream, first seen in Eggen (1958) and later detected in multiple surveys such as HIPPARCOS, RAVE, LAMOST, and Gaia (e.g., Dehnen 1998; Antoja et al. 2012; Liang et al. 2017; Gaia Collaboration 2018), is revealed as an elongated region in the $U V$-plane with a characteristic velocity moving away from the Galactic center, $U \approx-30-50 \mathrm{~km} \mathrm{~s}^{-1}$ and lagging behind the local standard of rest (LSR), $V \approx-60 \mathrm{~km} \mathrm{~s}^{-1}$. The hypothesis of the disruption of a cluster as its origin was discarded because of their spread in ages and metallicities (e.g., Famaey et al. 2005; Antoja et al. 2008; Bovy \& Hogg 2010). The stream was more likely formed through non-axisymmetries in the Galactic potential. In particular, the Hercules stream has for a long time been hypothesized to be caused by the effects of the outer Lindblad resonance (OLR) of the Galactic bar (Dehnen 2000; Fux 2001). More recently, alternative origins for Hercules have been proposed based on independent evidence for an extended slowly rotating bar in the Milky Way (Wegg et al. 2015; Portail et al. 2015) that would place the OLR too far beyond the solar neighborhood. This means that the Hercules stream could be related to the corotation resonance of the bar (Pérez-Villegas et al. 2017), the 4:1 OLR of a slowly rotating bar (Hunt \& Bovy 2018), and a combination of the effects of a slowly rotating bar and spiral arms (Hattori et al. 2019). This issue is far from being settled, and recent work still supports the original explanation (Hunt et al. 2018; Ramos et al. 2018; Fragkoudi et al. 2019).

While Hercules is the group with largest extension in velocity space, many studies have revealed substructures within it. For instance, two overdensities at approximately the same rotation velocity but at different Galactocentric radial velocity have been observed in Dehnen (1998) and Antoja et al. (2015). Recently, Gaia data showed the splitting of the Hercules stream into two or 
three branches of approximately constant Galactocentric radial velocity (Gaia Collaboration 2018; Ramos et al. 2018).

On the other hand, white dwarfs are long-living objects that represent the most common evolutionary remnants of lowand intermediate-mass stars, that is, stars with $M \lesssim 8 \sim 11 M_{\odot}$ (e.g., Siess 2007). Nuclear fusion reactions have ceased in white dwarf interiors; the pressure due to the degenerate electrons is responsible for preventing the gravitational collapse of these compact objects. White dwarfs are then subjected to a long process of gravothermal cooling, and their characteristics are reasonably well understood from a theoretical point of view; see, for instance, the review by Althaus et al. (2010). Hence, white dwarfs have been considered ideal candidates for constraining the age and formation history of the different Galactic components (e.g., Fontaine et al. 2001; Reid 2005; García-Berro \& Oswalt 2016).

However, to date, the use of white dwarfs as Galactic tracers of the dynamic evolution has been limited. In particular, only a few modest studies of the white dwarf population have been dedicated to the search of stellar streams in the solar neighborhood (e.g., Fuchs \& Dettbarn 2011) or to study the possible imprints of a merger episode in the Galactic disk (e.g., Torres et al. 2001). There are several reasons for this: first, we lack radial velocity measurements for white dwarfs unless optimum resolution spectra are available (e.g., Pauli et al. 2006; Anguiano et al. 2017). This is a consequence of the broadening of the Balmer spectral lines that arises from the high surface gravity that characterizes white dwarf atmospheres. Second, also due to this huge gravitational pull, metals are sunk in the deep interiors of white dwarf envelopes, which precludes associating a metallicity value with these objects. Finally, the number of white dwarfs that are identified in volume-limited samples is rather small.

Fortunately, the advent of large data surveys such as Gaia has dramatically increased the number of known white dwarfs, thus providing statistically significant large complete samples. Together with the potential of white dwarfs as cosmochronometers, this opens the door to using these objects as reliable tracers of the dynamic Galactic evolution.

In this Letter we aim to show the Hercules stream signature in the population of white dwarfs of the local neighborhood. Additionally, we aim to identify white dwarfs that belong to the Hercules stream and to derive a first approach to their age distribution.

\section{Gaia DR2 white dwarf 100 pc sample}

The second data release (DR2) of the Gaia mission has provided an unprecedented wealth of accurate astrometric and photometric information. In particular, nearly 260000 objects have been identified as white dwarf candidates (Gentile Fusillo et al. 2019) up to a few kiloparsec from the Sun. Moreover, Jiménez-Esteban et al. (2018) claimed that the largest and nearly complete Gaia white dwarf sample extends up to $100 \mathrm{pc}$ from the Sun and contains almost 18000 objects. For this sample and by means of applying innovative techniques based on a random forest machine-learning algorithm, Torres et al. (2019) have been able to classify the white dwarf population into its different Galactic components: thin and thick disk, and stellar halo. The final classified sample contains 12227 thin-disk, 1410 thickdisk, and nearly 100 halo white dwarf candidates. Heliocentric velocities are calculated in the standard Galactic coordinate velocity system, $(U, V, W)$, with $U$ positive toward the Galactic center, as derived from the five-parameter astrometric solution, $\left(\alpha, \delta, \varpi, \mu_{\alpha}^{*}, \mu_{\delta}\right)$, provided by the Gaia measurements.

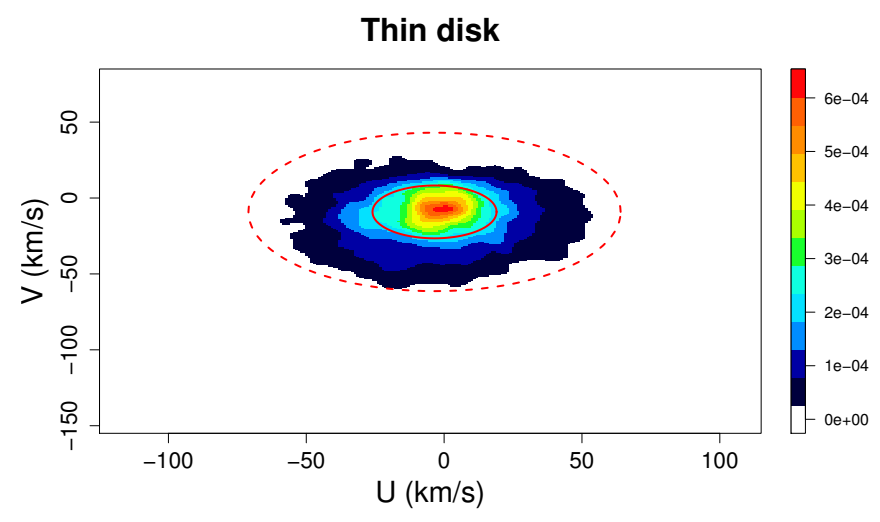

Thick disk

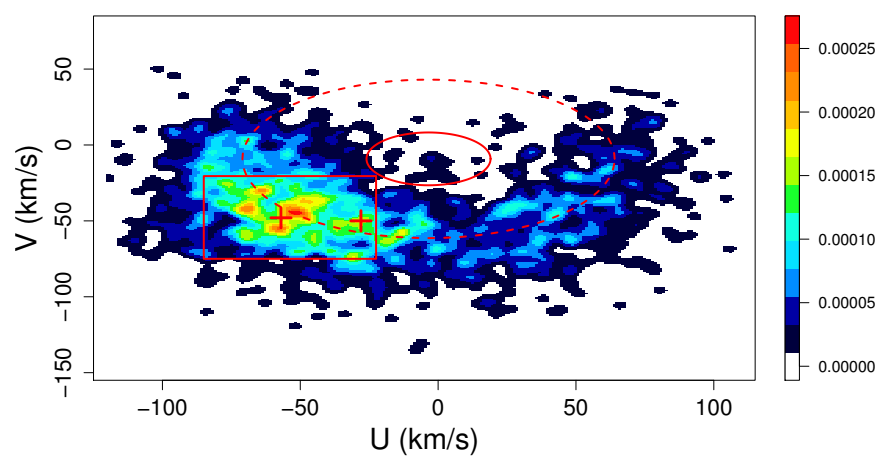

Fig. 1. $U V$ density diagram for the thin- (top panel) and thick-disk (bottom panel) white dwarf population within $100 \mathrm{pc}$ from the Sun. A clear overdensity is revealed in the thick-disk population (delimited by a red rectangle). For comparative purposes, we show the thin-disk dispersion ellipsoid for $1 \sigma$ (continuous line) and $3 \sigma$ (dashed line) levels corresponding to the thin-disk population (Torres et al. 2019), and we illustrate the number density (right scales) of white dwarf stars per $1 \times 1(\mathrm{~km} \mathrm{~s})^{-2}$. The location of the Hercules substreams from Antoja et al. (2012) is shown with red crosses. See text for details.

\section{Identifying white dwarfs in the Hercules stream}

The Hercules stream, as many other similar stellar streams, is first revealed as an overdensity of stars in the $U V$ plane (e.g., Dehnen 2000). Thus, as shown in Fig. 1, we started by analyzing the $U V$ space velocity plane by means of a density kernel estimation (Chen et al. 1997) for the 100 pc Gaia thin- and thick-disk white dwarf populations. No relevant feature departing from a Gaussian distribution appears when the thin-disk white dwarf population is depicted (top panel of Fig. 1). The $U$ distribution (after it is corrected for the solar motion) is reasonably symmetric: $53 \%$ to $47 \%$ of objects with $U>0$ and $U<0$, respectively, in the region with $V<0$. However, when the white dwarf thick-disk population is represented (bottom panel of Fig. 1), a clear overdensity is revealed that breaks the $U$ symmetry. Now, $68 \%$ of these objects present a negative $U$ velocity. This overdensity region (red rectangle), centered at around $(U, V)=(-55,-50) \mathrm{km} \mathrm{s}^{-1}$ and with an extension of $(\Delta U, \Delta V)=(60,50) \mathrm{km} \mathrm{s}^{-1}$, presents a number of objects per $\left(\mathrm{km} \mathrm{s}^{-1}\right)^{2}$ that is approximately three times larger than the average density. The so-called $U$-anomaly is thus clearly visible in the $U V$ plane for the thick-disk distribution.

It is worth mentioning that this feature is not a consequence of the lack of radial velocities in the white dwarf sample. Our Monte Carlo population synthesis analysis performed in Torres et al. (2019) revealed that the assumption of null radial 

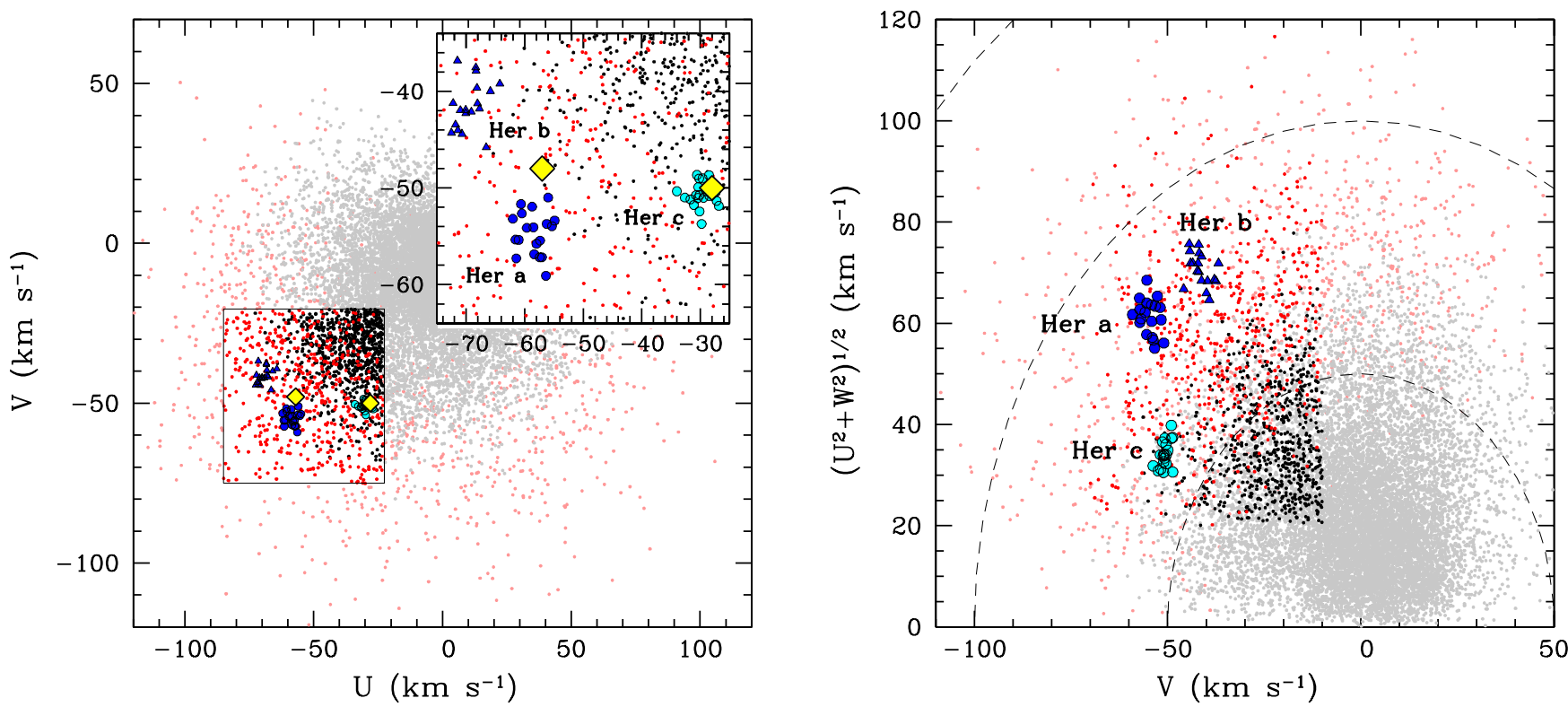

Fig. 2. UV diagram (left panel) and Toomre diagram (right panel) for the thin- and thick-disk white dwarf population (black and red dots, respectively, and we highlight with color those that belong to the selected overdensity region). We also plot the structures Her $a$ (blue circles), Her $b$ (blue triangles), and Her $c$ (cyan circles) that we found here. We show the location of the Hercules substreams from Antoja et al. (2012) in the $U V$ plane with yellow squares.

velocity implies a reduction of the speed moduli of the stars. In particular, the $(U, V)$ components are expected to be reduced by $(3.7 \pm 17,7.2 \pm 18) \mathrm{km} \mathrm{s}^{-1}$ and $(4.0 \pm 29,10.7 \pm 32) \mathrm{km} \mathrm{s}^{-1}$ on average for the thin- and thick-disk populations, respectively. However, this fact does not generate any asymmetry in their final component distributions. In the same sense, the incompleteness of the thick-disk white dwarf sample cannot be argued to be the cause of this peculiar overdensity. Thick-disk white dwarfs are extremely difficult to distinguish from thin-disk white dwarfs in low-speed stars. This is responsible for the characteristic halfmoon shape that appears in the thick-disk distribution, but again, it cannot justify the observed overdensity.

When any possible selection bias is discarded as its cause, the overdensity of white dwarfs found in the $U V$ plane is revealed as a kinematic feature different in nature from the thinor thick-disk populations. Moreover, the location within the $U V$ plane is in perfect agreement with previous identifications of the Hercules stream; see the bottom panel of Fig. 1. We can conclude then that the Hercules stream signature is present in the kinematics of the white dwarf population.

We now aim to identify white dwarfs that are genuine members of the Hercules stream. The loci of this stream within the $U V$ plane are superimposed on the standard population of thickdisk white dwarfs and also on high-speed thin-disk stars. For this reason, we need to extend our analysis in order to distinguish the different populations. Because of the lack of radial velocity and metallicity measurements, we must extract the maximum possible information contained in the tangential velocity of each white dwarf. To this end, the following procedure was carried out. First, we created a 5D space with the variables that are relevant for distinguishing stellar kinematic populations. In our case, the chosen variables were the $U$ and $V$ Galactic velocity components; the modulus or peculiar velocity of the star, $V_{\text {pec }} \equiv\left(U^{2}+V^{2}+W^{2}\right)^{1 / 2}$; the velocity perpendicular to the Galactic rotation as defined in the Toomre diagram, $V_{\text {Toomre }} \equiv\left(U^{2}+W^{2}\right)^{1 / 2}$; and $V_{\Delta E}=\left(U^{2}+2 V^{2}\right)^{1 / 2}$, which is derived from the integral of motion and is related to the eccentricity, $e$, of the orbit through $V_{\Delta E}=\sqrt{2} e v_{\mathrm{c}}$, where $v_{\mathrm{c}}$ is the Galactic velocity assuming a flat rotation model. This set of variables has been widely used in the search for stellar kinematic structures (e.g., Bensby et al. 2007; Fuchs \& Dettbarn 2011) and provides a complete set of dynamical properties.

Our second step was to apply a machine-learning technique in order to identify a genuine group of Hercules stream white dwarfs because it is expected that members of a kinematic structure share similar characteristics in our 5D space. Among the machine-learning techniques, several examples of unsupervised density-based clustering multipurpose methods that are widely used in astrophysics are available. The most popular are DBSCAN, HDBSCAN , and OPTICS (see, e.g., Cánovas et al. 2019, for a thorough description of the algorithms and references there in). Here we chose HDBSCAN, which represents an extension of DBSCAN and improves its performance by implementing a hierarchical clustering strategy. Very few hyperparameters (free parameters introduced by the user) are required by the HDBSCAN algorithm. We adopted a value of the hyperparameter mPts (minimum number of objects required to form a cluster) in the range 10-30 and a probability to belong to a cluster higher than $90 \%$. The first criterion ensures a minimum physical significance of the cluster, while the second guarantees that all members of a particular cluster share similar properties.

After we normalized our 5D space following a standard scaler and using a Euclidean metric, we applied the HDBSCAN algorithm to the $U V$ overdensity region (red rectangle in the bottom panel of Fig. 1) to our thin- plus thick-disk white dwarf population. The algorithm found four clusters or groups. The main group, formed by almost 400 objects, contains mainly field thin-disk white dwarfs that lie within the selected region. The other three groups, those that we are interested in, are formed each by almost 20 stars and reveal kinematic characteristics that are different from those of the thick- and thin-disk population. These three groups are shown in Fig. 2 along with the white dwarfs of the overdensity selected region for different combinations of our 5D space variable. The first of these three groups 
Table 1. Hercules substream locations.

\begin{tabular}{lcc}
\hline \hline Source & Substream & $(U, V) \mathrm{km} \mathrm{s}^{-1}$ \\
\hline This work & Her $a$ & $(-59 \pm 2,-55 \pm 3)$ \\
This work & Her $b$ & $(-69 \pm 2,-41 \pm 2)$ \\
This work & Her $c$ & $(-30 \pm 2,-51 \pm 1)$ \\
Antoja et al. (2012) & Her I & $(-57,-48)$ \\
Antoja et al. (2012) & Her II & $(-28,-50)$ \\
Bobylev \& Bajkova (2016) & Her I & $(-57,-48)$ \\
Bobylev \& Bajkova (2016) & Her II & $(-35,-55)$ \\
Ramos et al. (2018) & A8 & $\sim([-30,-70],-35)$ \\
Ramos et al. (2018) & A9 & $\sim([-50,+10],-50)$ \\
\hline
\end{tabular}

obtained by HDBSCAN (blue circles) is located in the $U V$ plane (left panel of Fig. 2) at $(U, V)=(-58.5,-54.7) \mathrm{km} \mathrm{s}^{-1}$ and contains 19 white dwarfs. The second group (blue triangles), close to the first one, is located at $(U, V)=(-69.3,-41.4) \mathrm{km} \mathrm{s}^{-1}$ and contains 18 objects. Finally, the third group (cyan circles) is formed by 20 stars centered at $(U, V)=(-29.9,-50.7) \mathrm{km} \mathrm{s}^{-1}$. We call these groups Hercules (Her) $a, b$ and $c$. The first relevant conclusion here is that these groups are in excellent agreement with the Hercules stream location found in the literature. Although the Hercules stream was initially discovered as a diffuse elongated region in the $U V$ space, later analyses claimed that the Hercules stream is formed by two substreams called Hercules I and II (Antoja et al. 2012; Bobylev \& Bajkova 2016). Recent studies based on several million FGK stars provided by the Gaia-DR2 analysis (Gaia Collaboration 2018) instead reveal Hercules substreams as thin-arch structures (Ramos et al. 2018; Li \& Shen 2019). Our groups Her $a$ and $b$ are in agreement with the Hercules I substructure, while our group Her $c$ perfectly matches Hercules II. On the other hand, our Her $b$ and $c$ are consistent with structure A9 and Her $a$ with A8 found in Ramos et al. (2018). A deeper study (including, e.g., radial velocities) is needed to ascertain the ultimately origin of our three groups. Meanwhile, we treated them separately. In Table 1 we summarize the results ${ }^{1}$.

It is worth mentioning that our Her $a$ structure is exclusively formed by thick-disk stars, while for Her $b$, all but one star $(5 \%)$ belong to the thick-disk population. Conversely, our Her $c$ substructure is formed by $13(65 \%)$ thin-disk and 7(35\%) thick-disk white dwarfs. These results are also in agreement with the spread in metallicities found in the Hercules stream (Hattori et al. 2019).

Although it might be argued that the effects of the bar are mostly constrained to the thin-disk population under the claim that orbits reaching high altitudes in the plane would not be that much perturbed, simulations have shown that the bar induces a Hercules-like structure on the thick disk comparable to that of the thin-disk. Thus, the existence of Hercules in the thick-disk population is, from the dynamical point of view, entirely plausible in the scenario of the bar resonant effects. The first observational evidence that the effects of the bar are also present in the thick disk was shown in Antoja et al. (2012), and more recently, Koppelman et al. (2018) have also identified an asymmetry in the $U$ velocity tail of the thick disk that could have the same origin.

\footnotetext{
1 A complete list of the white dwarfs belonging to each of the three groups identified in this work is available upon request.
}

\section{Age distribution estimation}

We performed a first estimation of the ages of the white dwarfs that are identified in the Hercules stream so far. Because of the lack of spectroscopic information, we adopted some assumptions. First, we assumed hydrogen-rich atmospheres (also known as DA white dwarfs) for all the stars in our sample. Second, we adopted a solar metallicity value, $Z=0.01$, for thin-disk white dwarfs. Although a small dispersion around the solar value is expected (e.g., Tononi et al. 2019), discrepancies in the age determinations are negligible when the final age distribution is binned in intervals of 1 Gyr. Similarly, a subsolar value, $Z=0.001$, was adopted for the thick-disk white dwarfs. From the parallax and photometry measurements provided by Gaia, we derived the absolute magnitude, $M_{\mathrm{G}}$, and the color $G_{\mathrm{BP}}-G_{\mathrm{RP}}$ for each star of our sample. After we adopted a set of cooling tracks (we used those of Althaus et al. 2015, Camisassa et al. 2016, 2019, which encompass a full range of masses and progenitor metallicities and provide a full set of self-consistent cooling sequences), we obtained a robust estimate of the total age (white dwarf cooling time plus progenitor lifetime) for each object of our Hercules sample. Objects with masses below $0.53 M_{\odot}$ were discarded because they probably belong to binary systems in which a common-envelope episode has occurred, and their ages can therefore not be traced back (e.g., Rebassa-Mansergas et al. 2011).

In Fig. 3 we show the age distribution for the white dwarfs that we identified in the Her $a, b$, and $c$ streams and also the distribution belonging to the three streams together (right panel). Additionally, a Kolmogorov-Smirnov (K-S) test between the age distributions reveals that the null hypothesis (i.e., that two distributions have the same origin) cannot be rejected with a probability, $P$, of $\sim 0.70$, when Her $a$ and $c$ or Her $b$ and $c$ are compared. However, when Her $a$ and $b$ are compared, the probability to have the same origin decreases to $P=0.44$. Our K-S test also shows (see Fig. 3) that Her $a$ and Her $c$ are consistent with a normal distribution $(P=0.94$ and $P=0.86)$, while the age distribution of Her $c$ is probably not normally distributed $(P=0.17)$, but consistent with a log-normal distribution $(P=0.81)$. When the entire population of Hercules white dwarfs is considered, a log-normal distribution that peaked $4 \mathrm{Gyr}$ in the past with a secondary slight peak at $8 \mathrm{Gyr}$ extended to old ages seems a reasonable fit $(P=0.61)$, disregarding a doubleGaussian distribution $(P=0.13)$. Although these distributions should be understood as preliminary guesses, the extended ages found here are in agreement with the dynamical origin of the Hercules stream, disregarding any cluster disruption hypothesis.

\section{Conclusions}

We have revealed the imprint of the Hercules stream in the space velocity of white dwarfs in the solar neighborhood. In particular, we analyzed the recent Gaia white dwarf population, which presents a great wealth of accurate photometric and astrometric data and a nearly complete sample within $100 \mathrm{pc}$. The analysis of the white dwarf $U V$ plane clearly shows an overdensity of objects in agreement with previous observed signatures of the Hercules stream in main-sequence stars by other surveys such as HIPPARCOS, RAVE, or LAMOST.

Taking advantage of an advanced hierarchical clustering algorithm, HDBSCAN, which we applied to a 5D space of kinematic variables, we were able to identify white dwarfs that are genuine members of the Hercules stream. We identified three main substreams, Her $a, b$, and $c$, located at $(U, V)=$ $(-59,-55) \mathrm{km} \mathrm{s}^{-1},(-69,-41) \mathrm{km} \mathrm{s}^{-1}$, and $(-30,-51) \mathrm{km} \mathrm{s}^{-1}$, 

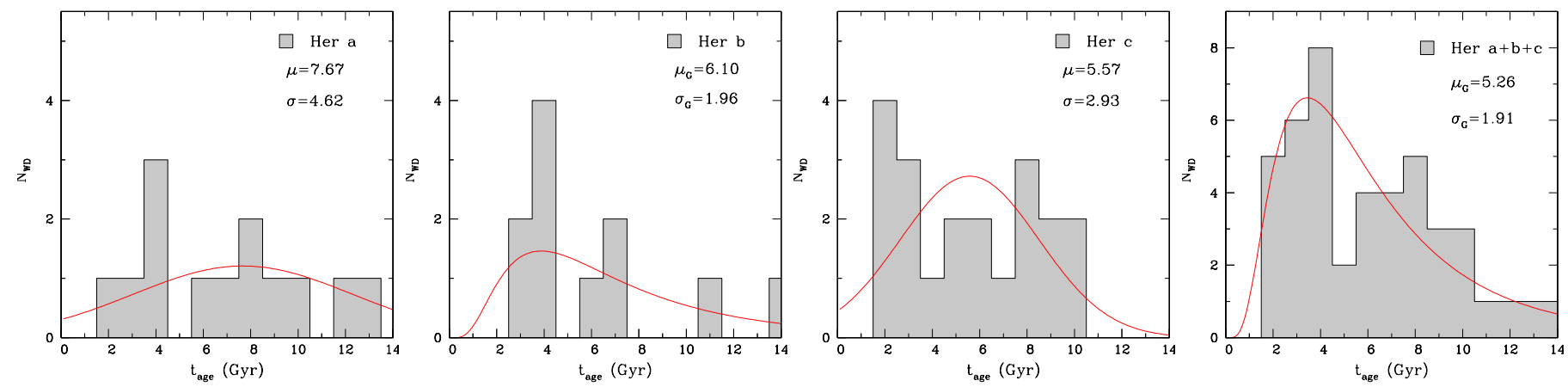

Fig. 3. Age distribution for each of the three Hercules substreams identified in this work and for all of them considered together (right panel). The substream Her $b$ presents a maximum at around $4 \mathrm{Gyr}$ with an extended long tail up to very old ages. Her $a$ is similar, but with a less clear peak. Substream Her $c$ appears as a more uniform distribution between 2 and $10 \mathrm{Gyr}$. We also plot as red lines analytical normal fits for Her $a$ and $c$ and a $\log$-normal fit for Her $c$ and the entire distribution. The corresponding mean $\mu$ and deviation $\sigma$, the geometric mean $\mu_{G}$, and the multiplicative deviation $\sigma_{G}$ are depicted in each panel.

respectively. The first two are mainly formed by thick-disk white dwarfs. The third is a mixture of $65: 35 \%$ of thin- and thick-disk white dwarfs. Moreover, a first guess of their age distribution shows that Her $b$ presents a maximum at $4 \mathrm{Gyr}$ ago and extends to very old ages, similar to Her $a$. However, Her $c$ depicts a more uniform distribution of objects between 2 and $10 \mathrm{Gyr}$. Although the nature and origin of the Hercules stream still remain opens questions, the Hercules white dwarf sample identified in this work can provide very valuable information for clarifying these questions.

Acknowledgements. This work was partially supported by the MINECO grant AYA2017-86274-P and the Ramón y Cajal programme RYC-2016-20254, by the AGAUR, and by grant G149 from University of La Plata.

\section{References}

Althaus, L. G., Córsico, A. H., Isern, J., \& García-Berro, E. 2010, A\&ARv, 18, 471

Althaus, L. G., Camisassa, M. E., Miller Bertolami, M. M., Córsico, A. H., \& García-Berro, E. 2015, A\&A, 576, A9

Anguiano, B., Rebassa-Mansergas, A., García-Berro, E., et al. 2017, MNRAS, 469,2102

Antoja, T., Figueras, F., Fernández, D., \& Torra, J. 2008, A\&A, 490, 135

Antoja, T., Figueras, F., Torra, J., Valenzuela, O., \& Pichardo, B. 2010, in Lecture Notes and Essays in Astrophysics, 4, 13

Antoja, T., Helmi, A., Bienayme, O., et al. 2012, MNRAS, 426, L1

Antoja, T., Monari, G., Helmi, A., et al. 2015, ApJ, 800, L32

Bensby, T., Oey, M. S., Feltzing, S., \& Gustafsson, B. 2007, ApJ, 655, L89

Bobylev, V. V., \& Bajkova, A. T. 2016, Astron. Lett., 42, 90

Bovy, J., \& Hogg, D. W. 2010, ApJ, 717, 617

Camisassa, M. E., Althaus, L. G., Córsico, A. H., et al. 2016, ApJ, 823, 158

Camisassa, M. E., Althaus, L. G., Córsico, A. H., et al. 2019, A\&A, 625, A87
Cánovas, H., Cantero, C., Cieza, L., et al. 2019, A\&A, 626, A80

Chen, B., Asiain, R., Figueras, F., \& Torra, J. 1997, A\&A, 318, 29

Dehnen, W. 1998, AJ, 115, 2384

Dehnen, W. 2000, AJ, 119, 800

Eggen, O. J. 1958, MNRAS, 118, 154

Famaey, B., Jorissen, A., Luri, X., et al. 2005, A\&A, 430, 165

Fontaine, G., Brassard, P., \& Bergeron, P. 2001, PASP, 113, 409

Fragkoudi, F., Katz, D., Trick, W., et al. 2019, MNRAS, 488, 3324

Fuchs, B., \& Dettbarn, C. 2011, AJ, 141, 5

Fux, R. 2001, A\&A, 373, 511

Gaia Collaboration (Katz, D., et al.) 2018, A\&A, 616, A11

García-Berro, E., \& Oswalt, T. D. 2016, New A Rev., 72, 1

Gentile Fusillo, N. P., Tremblay, P.-E., Gänsicke, B. T., et al. 2019, MNRAS, 482, 4570

Hattori, K., Gouda, N., Tagawa, H., et al. 2019, MNRAS, 484, 4540

Hunt, J. A. S., \& Bovy, J. 2018, MNRAS, 477, 3945

Hunt, J. A. S., Bovy, J., Pérez-Villegas, A., et al. 2018, MNRAS, 474, 95

Jiménez-Esteban, F. M., Torres, S., Rebassa-Mansergas, A., et al. 2018, MNRAS, 480, 4505

Koppelman, H., Helmi, A., \& Veljanoski, J. 2018, ApJ, 860, L11

Li, Z. Y., \& Shen, J. 2019, ArXiv e-prints [arXiv:1904 .03314]

Liang, X. L., Zhao, J. K., Oswalt, T. D., et al. 2017, ApJ, 844, 152

Pauli, E.-M., Napiwotzki, R., Heber, U., Altmann, M., \& Odenkirchen, M. 2006, A\&A, 447, 173

Pérez-Villegas, A., Portail, M., Wegg, C., \& Gerhard, O. 2017, ApJ, 840, L2

Portail, M., Wegg, C., Gerhard, O., \& Martinez-Valpuesta, I. 2015, MNRAS, 448, 713

Ramos, P., Antoja, T., \& Figueras, F. 2018, A\&A, 619, A72

Rebassa-Mansergas, A., Nebot Gómez-Morán, A., Schreiber, M. R., Girven, J., \& Gänsicke, B. T. 2011, MNRAS, 413, 112

Reid, I. N. 2005, ARA\&A, 43, 247

Siess, L. 2007, A\&A, 476, 893

Tononi, J., Torres, S., García-Berro, E., et al. 2019, A\&A, 628, A52

Torres, S., García-Berro, E., Burkert, A., \& Isern, J. 2001, MNRAS, 328, 492

Torres, S., Cantero, C., Rebassa-Mansergas, A., et al. 2019, MNRAS, 485, 5573

Wegg, C., Gerhard, O., \& Portail, M. 2015, MNRAS, 450, 4050 